\title{
Wheelchair Fall Prevention System Data Device with Airport and Public Facilities
}

\author{
Seung-Hun $\mathrm{Kim}^{1}$, Min-Seok $\mathrm{Jie}^{2}$ and Won-Hyuck Choi ${ }^{3 *}$ \\ ${ }^{1}$ Hanseo University, Department of Aeronautical System Engineering \\ 236-49, Gomseom-ro, Nam-myeon, Taean-gun, \\ 32158 Chungcheongnam-do, Republic of Korea \\ kimsh014@gmail.com \\ ${ }^{2, * 3}$ Hanseo University, Department of Avionics Engineering \\ 236-49, Gomseom-ro, Nam-myeon, Taean-gun, \\ 32158 Chungcheongnam-do, Republic of Korea \\ \{choiwh,jiems\}@hanseo.ac.kr
}

\begin{abstract}
The structure of the wheelchair is primarily, is fallen front, it is possible to safety accident of the passenger occurs. Terrain, if the wheelchair posture is not correct in accordance with the feature, it is impossible to ensure the safety of the rider. Wheelchair passenger using an acceleration sensor in order to safely use, was to be able to display the value of the ACR in real time. In this study, we use the algorithm of SDD(Safety Data Device) was developed it is possible to output and early detection of the wheelchair of attitude data.
\end{abstract}

Keywords: Wheelchair, Safety data device, Sensor Module, Accelerometer.

\section{Introduction}

Korea's aged population over 65 is expected to increase 2.3 times in 2030 and more than 3 times in 2060 compared to 2010. Unlike in the past, these aging societies have become a hot topic for their leisure activities due to the development of medical technology and the improvement of quality of life. In addition, the quality of the people with disabilities is receiving social attention as much as the elderly people are interested in. Medical devices such as wheelchairs are increasingly interested in medical devices that can complement the uncomfortable body functions and disabilities of older people and the disabled with the increase in the elderly population. Based on these social issues, the welfare industry activated in some welfare advanced countries has been activated in Korea, and the market size of the industry such as risk / safety facilities, medical / health, leisure / entertainment, It is increasing. Currently, wheelchairs are medical devices that are used for the purpose of transporting disabled persons who are not free of legs due to special diseases or accidents, and they are a typical pedometer that provides convenience for people such as elderly people who are uncomfortable to move.

For this reason, users of wheelchairs also increased. There are also a lot of wheelchair accidents. In the case of wheelchair related accidents in the CISS for consumers in Korea Consumer Agency, there is a tendency for the wheelchair users to fall due to immaturity, or a lack of strength in climbing the ramp, Wheelchair fall accidents such as falls on an obstacle, fractures in arms and legs, and a concussion overturned in a wheelchair constitute a large part of wheelchair fall accidents.

An example of such an unstable posture is the largest factor for a user who falls on a wheelchair. When a wheelchair passes a ramp, or passes an uneven road, the user's posture and wheelchair become unstable, leading to a fall. Therefore, we extract safety 
data using acceleration sensor and gyro sensor, and propose an algorithm that can detect early fall risk of elderly people using wheelchair. As soon as the danger is detected and the alarm is sounded, the occupant can be more careful and help the people around him.

In this paper, we developed an SDD (Safety Data Device) system that can detect the danger and prevent accidents by using the 3-axg value. In the following two chapters, we will study the research related to this development. In Chapter 3, we describe the developed system. Section 4 describes experimental methods and results and concludes in Section 5.

\section{Structure and Safety Sensor of Electric Card and Wheelchair}

\subsection{Structure of Wheelchair}

A wheelchair with a safety sensor attached to it will use a manual wheelchair. The motorized wheelchair includes a motorized braking device that can be braked by the user's will, and the speed of the motorized wheelchair is limited when the experiment is carried out. The speed and braking system were free and passive wheelchairs were used frequently. [Figure 1] shows various variables such as the center of gravity of a user in a wheelchair as a passenger wheelchair, the distance between the main wheel and the center of gravity, and the difference in height. The wheelchair's motion is determined by the two rear wheel rotations determined by the user's steering, $\theta_{R}$ and $\theta_{L}$, and if the rotation amount is different, the inertia is reduced. In this figure, $\mathrm{b}$ is the width of the car, and e is the vertical distance from the car width center to the center of gravity. The motion state of the wheelchair can be accurately described by $\Phi_{P}$ representing the direction of the wheelchair's heading, and coordinates representing the position of the center of gravity, $\mathrm{P}$, $\chi_{P}$ and $x_{P}$. The relationship between these variables is determined by Eqs. (1) - (4), and the following assumptions apply to the derivation of these equations.

$$
\begin{aligned}
& \dot{x}_{p}(t)=v(t) \sin \phi_{P} \\
& \dot{y}_{p}(t)=v(t) \cos \phi_{p} \\
& \phi_{p}(t)=\frac{\dot{\theta}_{R}-\dot{\theta}_{L}}{b} r=\frac{\omega_{R}-\omega_{L}}{b} r \\
& \mathcal{V}(\mathrm{t})=\frac{\omega_{R}-\omega_{L}}{2} r
\end{aligned}
$$

In the above equation, $\mathcal{V}(\mathrm{t})$ represents the speed of the wheelchair, $\omega_{R}$ and $\omega_{L}$ represent the rotational speed of the left and right rear wheels, and $r$ represents the radius of the rear wheel, respectively. Depending on the command signals $\theta_{R}$ and $\theta_{L}$ generated by the user's steering, the rear wheels have rotational angular velocities, $\omega_{R}$ and $\omega_{L}$. The velocity of the wheelchair is determined by Equation (4), from which all values of the state variables $\left(x_{P}, \mathcal{Y}_{P}, \phi_{P}\right)$ representing the motion of the wheelchair are determined. 


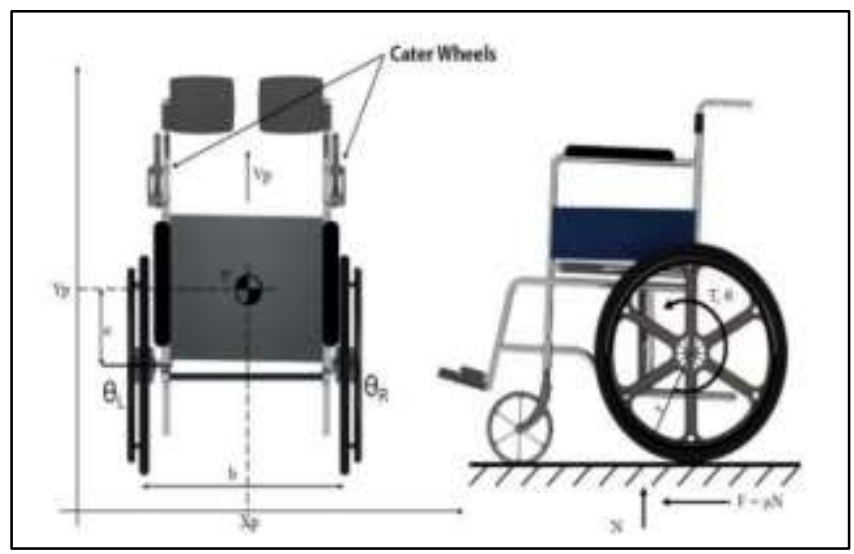

Figure 1. Wheel Body Structure

\subsection{Detection Sensor}

Those with low cognitive abilities use a wheelchair after a long practice session to use the wheelchair. However, since not all terrains are uneven, unexpected terrain can overturn a wheelchair, or cause a user to be injured by a large impact. We want to avoid situations where the wheelchair is overturned or injured by a big shock due to the incline, uphill, downhill, or passing speed. It also helps to use the wheelchair more safely by recognizing the danger in uneven terrain.

The first is to measure the same value as if the gyro sensor was tilted backward due to the law of inertia when the wheelchair started momentarily.

The second is used to measure the angle based on the gyro sensor. First, Equation 5 is a formula for calculating the angle using a gyro sensor, where $\mathrm{G}$ is the angular velocity, $\mathrm{dt}$ is the instantaneous change in time, $\mathrm{D}$ is the angle, and $\mathrm{C}$ is the integral constant.

$\int G \cdot d t=D+C$

Using the above equation, the angular value can be obtained, but the accumulation occurs over time and the correct angular value is not measured.

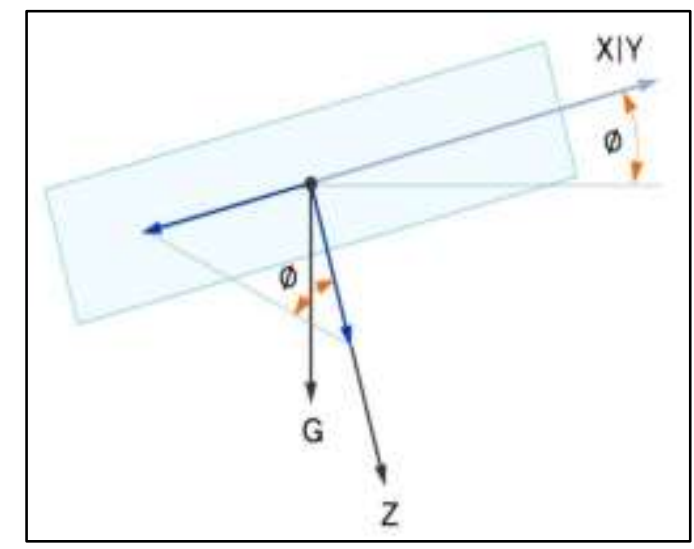

Figure 2. Acceleration Sensor Structure

Generally, the acceleration sensor represents gravitational acceleration values of the $\mathrm{X}$, $\mathrm{Y}$, and $\mathrm{Z}$ axes, which are gravitational acceleration values. However, to obtain the rotation angle, we obtain the trigonometric formulas as Eqs. (6)and (7) 


$$
\begin{aligned}
& \Phi=\tan ^{-1}(z / x) \\
& \Phi=\tan ^{-1}(z / y)
\end{aligned}
$$

As shown in [Figure 2], the left speed is the counterclockwise direction of the gyro sensor angle, and the acceleration sensor has an angle of four directions, so the sign of the whole is put in the whole. The reference angle is based on the plane perpendicular to the ground plane, and the angles of the acceleration sensor are based on the ground plane and the horizontal plane. Therefore, subtract $90^{\circ}(\pi / 2)$ from the angle calculated by the acceleration sensor. Therefore, if we take these two points into account, we can obtain Eqs. (8) and (9).

$$
\begin{aligned}
& \Phi=-\tan ^{-1}\left(\frac{z}{x}\right)-\frac{\pi}{2} \\
& \Phi=-\tan ^{-1}\left(\frac{z}{y}\right)-\frac{\pi}{2}
\end{aligned}
$$

Using the above equation, the angle is output, but the value can not be measured accurately due to noise. If you use the acceleration sensor only, the sensor value will be output quickly, but the correct value will not be output due to noise and accumulation of error value. When the complementary filter is used, the value output from the sensor is output late due to the correction process. When the Kalman filter is used, the corrected value is output more accurately than the complementary filter, but the value is output somewhat later. In this paper, one acceleration sensor, complementary filter, and Kalman filter were used.

When used in a wheelchair, it is used indoors as well as indoors. When used indoors, it has low thresholds, low obstacles on the floor, and obstacles that the user has not seen. Outdoors, it can be used on asphalt, sidewalk blocks, unpacked roads, and there are many obstacles in the outdoors such as stones and garbage. In addition, there will be worse conditions than the interior, such as the gap between the ramp and the sidewalk. Unlike an automobile, a passenger wheelchair, which is not an electric wheelchair, is not equipped with a suspension, ie, a shock absorber, which is very dangerous in such a small situation. Therefore, in order to enable the user to safely use the wheelchair, the user sees the risk in real time from the mounted sensor, obtains the 3-axg value according to the angle and speed, and detects the danger from the safety data device.

\section{Safety Data Device (SDD) Algorithm}

\subsection{3-axg(3-axis based on the average)}

3 -axg is a statistical measure of the magnitude of the changing value. This is especially useful when variables come in both negative and positive values, such as sine functions. It can be computed for a series of finite values or for a continuously varying function, and is the square root of the mean of the squares of the values as the name implies. Amplitude is an 'additional' unit in addition to the 'main unit' of the displacement-velocity-acceleration, which must be known or added to the actual value (3-axg).

The reason for expressing the 3-axis based on the average is that the concept of the area is deeper in relation to the energy than in the pointed graph in the case of speed or acceleration, It is defined as engineering interpretation and grammar standard (ISO) that there is deep relation.

$$
\mathrm{A} \cdot \mathrm{C} \cdot \mathrm{R}=\sqrt{\frac{\left(x_{t_{1}}-x_{t_{2}}\right)^{2}+\left(y_{t_{1}}-y_{t_{2}}\right)^{2}+\left(z_{t_{1}}-z_{t_{2}}\right)^{2}}{3}}
$$


The attitude control RMS (ACR) is a statistical measure of the magnitude of the changing value. This is especially useful when variables come in both negative and positive values, such as sine functions. It can be computed for a series of finite values or for a continuously varying function, and is the square root of the mean of the squares of the values as the name implies.

The reference position may be, for example, the center line of an axis or the midpoint between the highest position and the lowest position of a swinging object. Vibration refers to an alternating (repetitive) motion pattern based on a certain reference position, and vibrations can be moved only when there is a first force or a continuous force, so that expressing amplitude refers to a force or a variable related to force do. The amplitude of vibration is expressed in terms of displacement, velocity, and acceleration and should be applied differently depending on the object being vibrated or the object being evaluated.

When the owner moves, the ACR value changes. The larger the ACR value is, the higher the ACR value is. Especially, in the case of fall, the value changes suddenly, so the fall can be recognized only by measuring the acceleration value. However, the ACR value is an abrupt change in the output of a wheelchair fall, and it can not be known which direction the fall occurred when the fall occurred.

\subsection{SDD (Safety Data Device) Algorithm}

The gyro sensor measures the acceleration of the wheelchair to determine whether it is an accurate hazard, and the gyro sensor judges the inclination of the wheelchair to reach the range of influence of the wheelchair or to detect the fall of the wheelchair. The 3-axg value is output in real time. The 3 -axg value is calculated using the equation, and $\mathrm{x}, \mathrm{y}$, and $\mathrm{z}$ are the respective axes, and the 3-axg value is changed. When the motion is large and rapidly changed, the 3-axg value is high. Especially in the case of falls, the risk can be recognized because the value changes rapidly. By using the amount of change of the $X$, $\mathrm{Y}$, and $\mathrm{Z}$ axes, it is possible to know in which direction the wheelchair has fallen. It can be seen whether the value of the $X$ axis has fallen to the left or right depending on the value of 0 based on the value of 0 , and whether the $Y$ axis has fallen forward or backward based on the value of 0 as in the case above Can be judged.

The safety data device (SDD) algorithm checks the acceleration and slope changes of the wheelchair and then compares the 3-axg value of acceleration with the 3-axg value of the gyro through 3-axg value. If the 3 -axg threshold value is exceeded, 


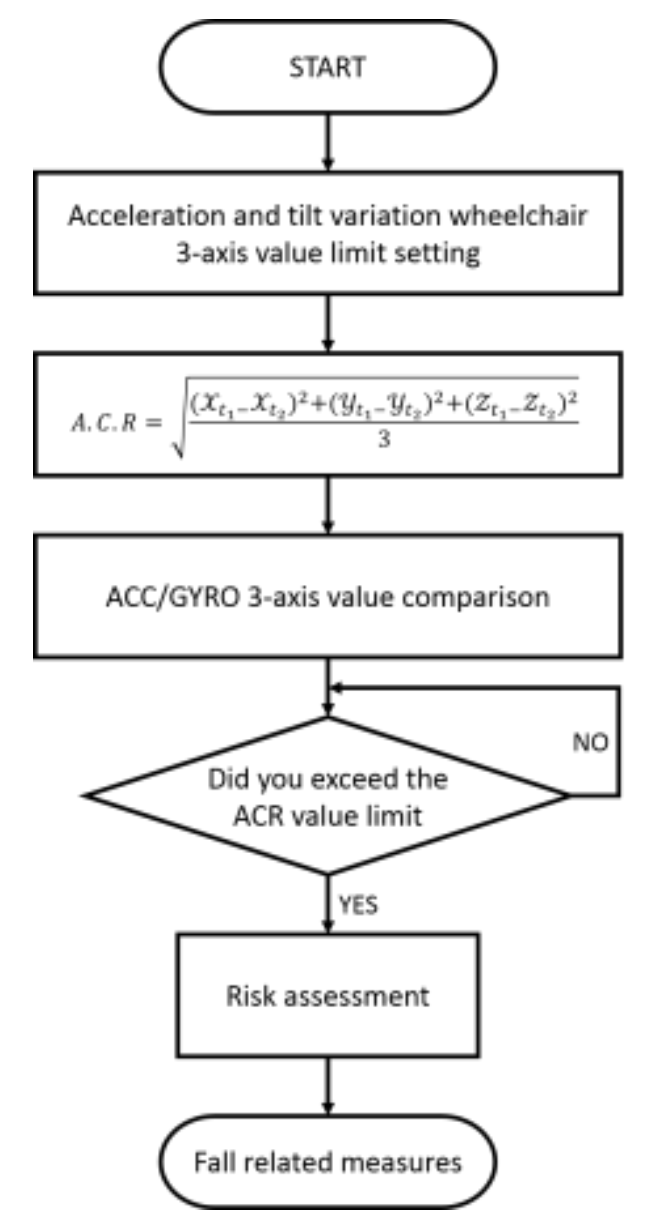

Figure 3. SDD Algorithm Flow Chart

\subsection{SDD (Safety Data Device) Extraction Algorithm}

When the acceleration 3-axg value is 400 or more and the slope 3 -axg value is 200 or more, it is judged as a risk. Acceleration 3-axg values varied somewhat according to different risk-assessment methods, but the slope 3-axg values remained constant above 200.

The problem here is that there is an ambiguous section in which it is difficult to distinguish whether the movement occurred in a certain interval between daily life and the danger zone. The range is the acceleration 3 -axg range from 300 to 400 . This problem is caused by the difference in the behavior that falls and the difference in the behavior of individual by sex and age group. To solve these problems, we provide a universal riskbased 3-axg threshold value that is appropriate for the user's age. When a sensorembedded device detects a danger, it receives feedback from the user and adjusts the threshold value. It develops into a combined fall detection system. That is why you get feedback feedback from users. 


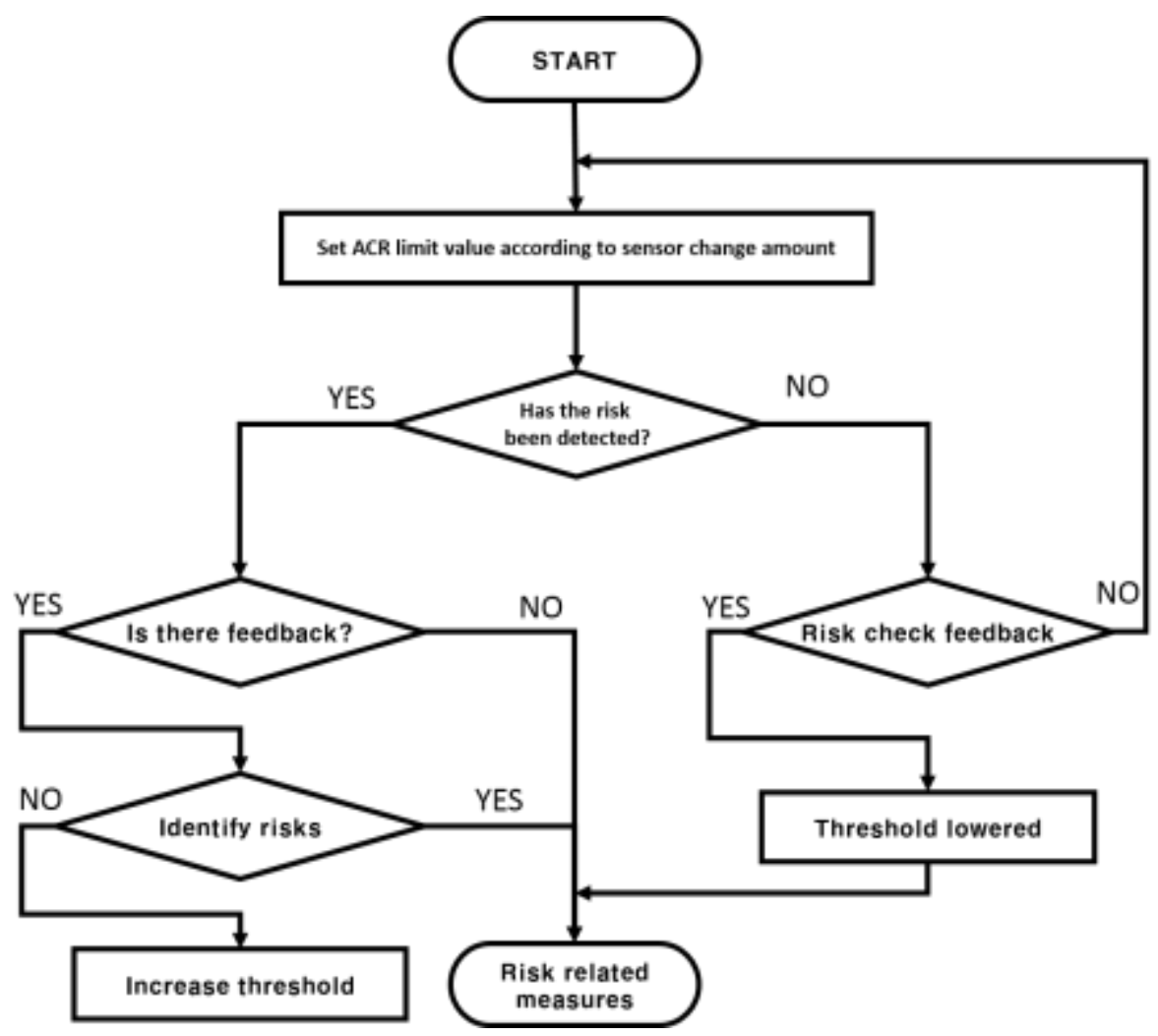

Figure 4. SSD Extraction Algorithm

\section{Algorithm Development for Safe Data Generation}

\subsection{Development of Safety Data Extraction Algorithm from Collected Sensor Data}

- The ACR detection method of the SDD mounted on the wheelchair proceeds in the following order.

- SDD Acceleration Sensor Data Collection

- Converting the collected data into gravitational acceleration data

- Calculation of $\mathrm{X}$-axis and $\mathrm{Y}$-axis tilt angles using converted gravity acceleration data

- Calculation of ACR value by using ACR function for X-axis and Y-axis inclination angle values

- Save the ACR value and repeat it in the same way

That is, in order to detect falling data using an acceleration sensor in a wheelchair, the ACR value is calculated using the slope value of the $\mathrm{X}$-axis and the slope value of the Yaxis. 


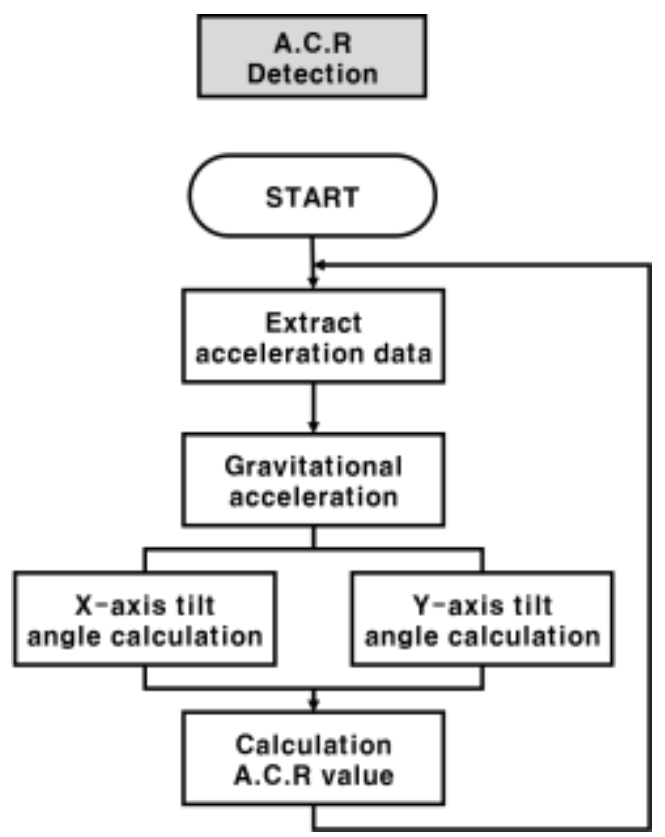

Figure 5. ACR Calculation using Acceleration Sensor

Data are collected and analyzed to determine the ACR threshold for fall time to specify the ACR threshold at the time of fall.

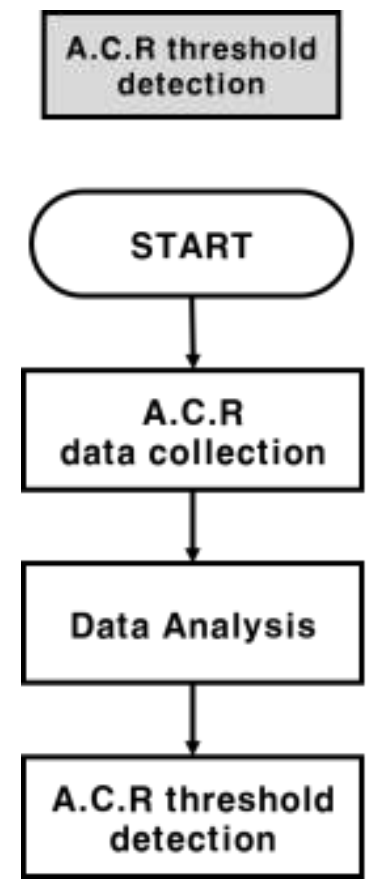

Figure 6. ACR Limit Value Detection Flow Chart

In order to precisely grasp the fall section, it is possible to compare the waveforms of the gravitational acceleration sensor in the stopped state and the gravitational acceleration sensor in the moving state to determine the fall section. 


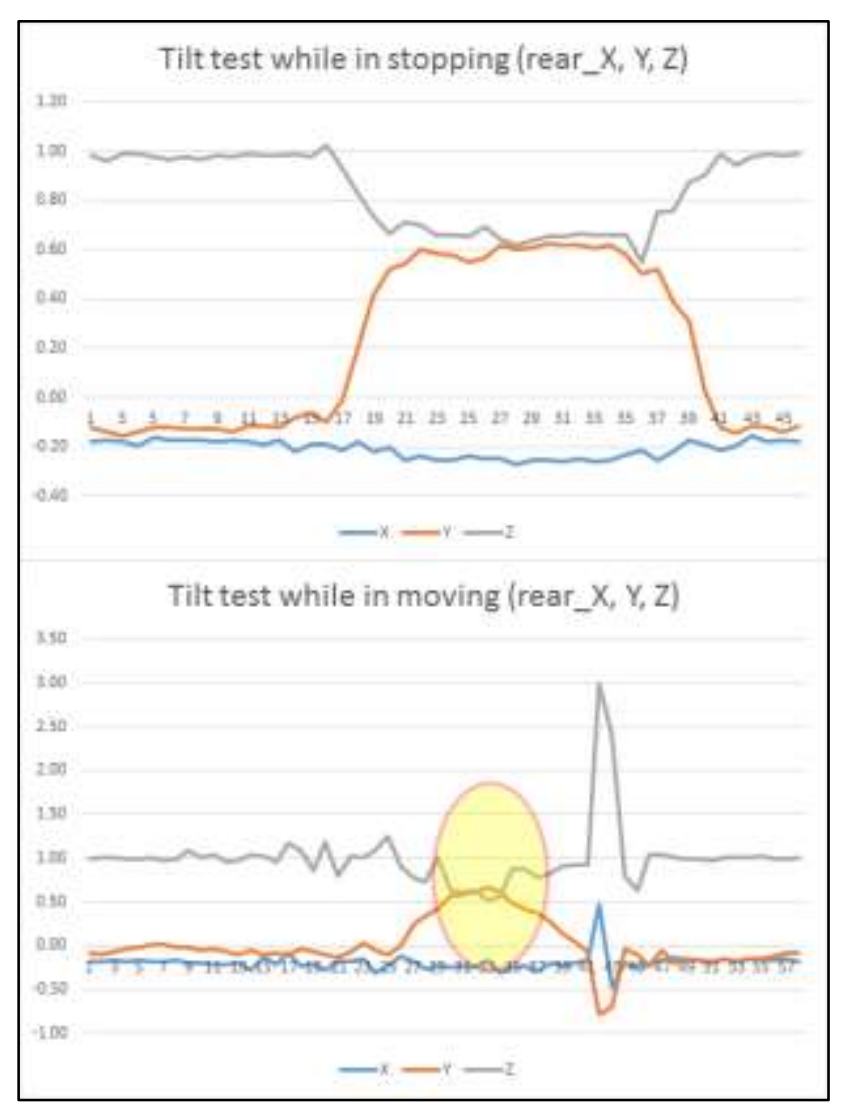

Figure 7. Accelerometer Tilt Test

As a result of the tilting test in the stopped state, it can be seen that the values of the Yaxis and the Z-axis change greatly. When we look at the graph, we can see that SDD is tilted forward at a high speed. If we look at the slope value during the movement, it is difficult to discriminate the value of $\mathrm{Z}$ axis due to frequent vibration, but the change amount of $Y$ axis value can be discriminated. It can be seen that the vibration of the wheelchair affects the $\mathrm{Z}$ axis while the wheelchair is moving and that the $\mathrm{Y}$ axis has a positive value when the wheelchair is tilted forward. Calculate the ACR value using the value of 3-axg to determine the danger zone.

The following figure shows the ACR value for that interval.

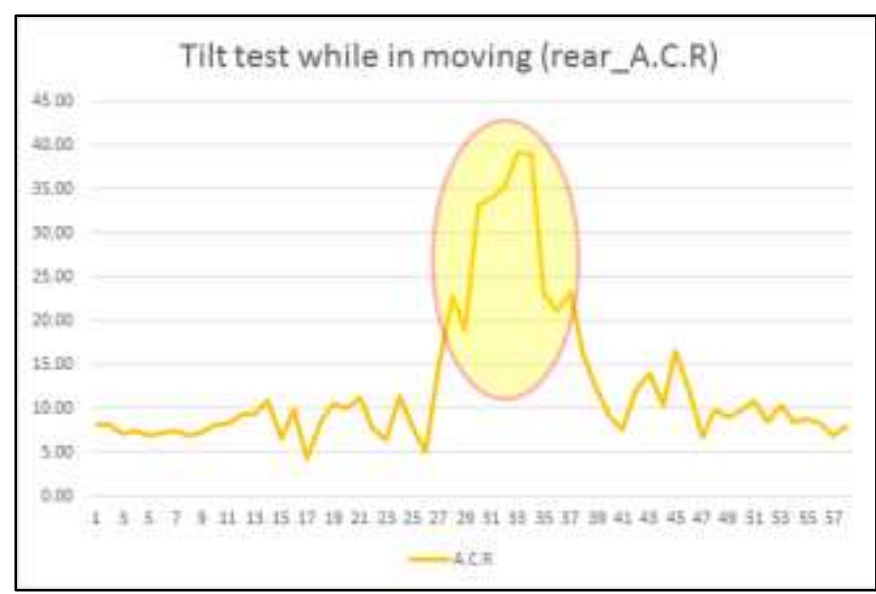

Figure 8. ACR Value of Fall Section 
In the case of Fig. 8, the ACR value is obtained using the 3-axg value. As shown above, we can see that the ACR value changes abruptly in one section. You can see the changes in the wheelchair when you look at the graph, but you can not tell exactly whether the passengers were aware of the danger or not. For that reason, directing a dangerous situation in many cases, the direct $175 \mathrm{~cm}$. A sturdy man weighing $70 \mathrm{~kg}$ is boarded directly to output the ACR value.

Figure 9 shows an algorithm for determining whether an actual fall occurs using the calculated ACR limit value. The acceleration sensor reads the 3-axg value in units of 100 $\mathrm{ms}$ and sends a fall risk message to the server when the ACR threshold is exceeded.

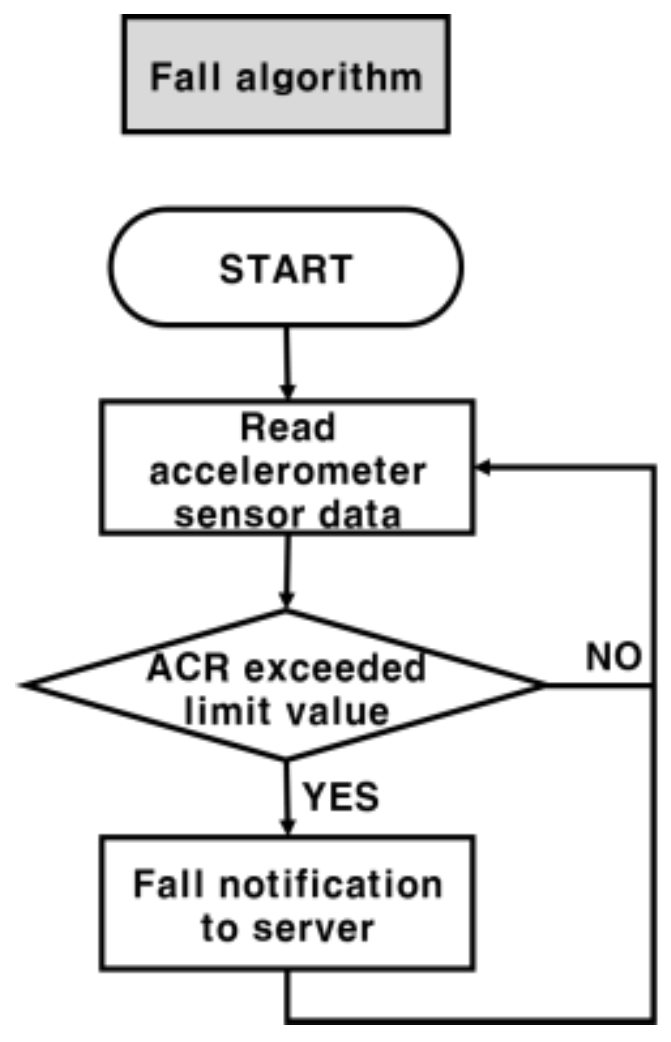

Figure 9. Fall Algorithm Flow Chart

\subsection{Simulation Results for Development Algorithm}

The relieving device uses the fall awareness algorithm to determine whether or not a fall has occurred, and transmits a fall event to the server when the fall occurs. Prior to the interworking test with the server, which is the result of the task execution, a demonstration program capable of receiving the data of the relieved device was developed and tested. The secure device and the demonstration program send and receive data through TCP / IP server and client structure.

The relieving device transmits the acceleration sensor value, the ACR value for the gradient calculated using the acceleration sensor, and the ultrasonic sensor value to the demonstration program. In addition, this sensor detects fall and transmits a warning message to the demo program.

If the SDD is mounted directly on the wheelchair, it is impossible to know whether the wheelchair is normally level or not, and it is difficult to judge the danger because the SDD value is not outputted normally even though the area is uneven. For this reason, we have confirmed that the SDD data is normally received by tilting the data based on a horizontal desk. 
As shown in the following figure 10, when the data of SDD is viewed, it can be confirmed that each axis of $\mathrm{X}, \mathrm{Y}, \mathrm{Z}$ and $\mathrm{ACR}$ value are constantly inputted.

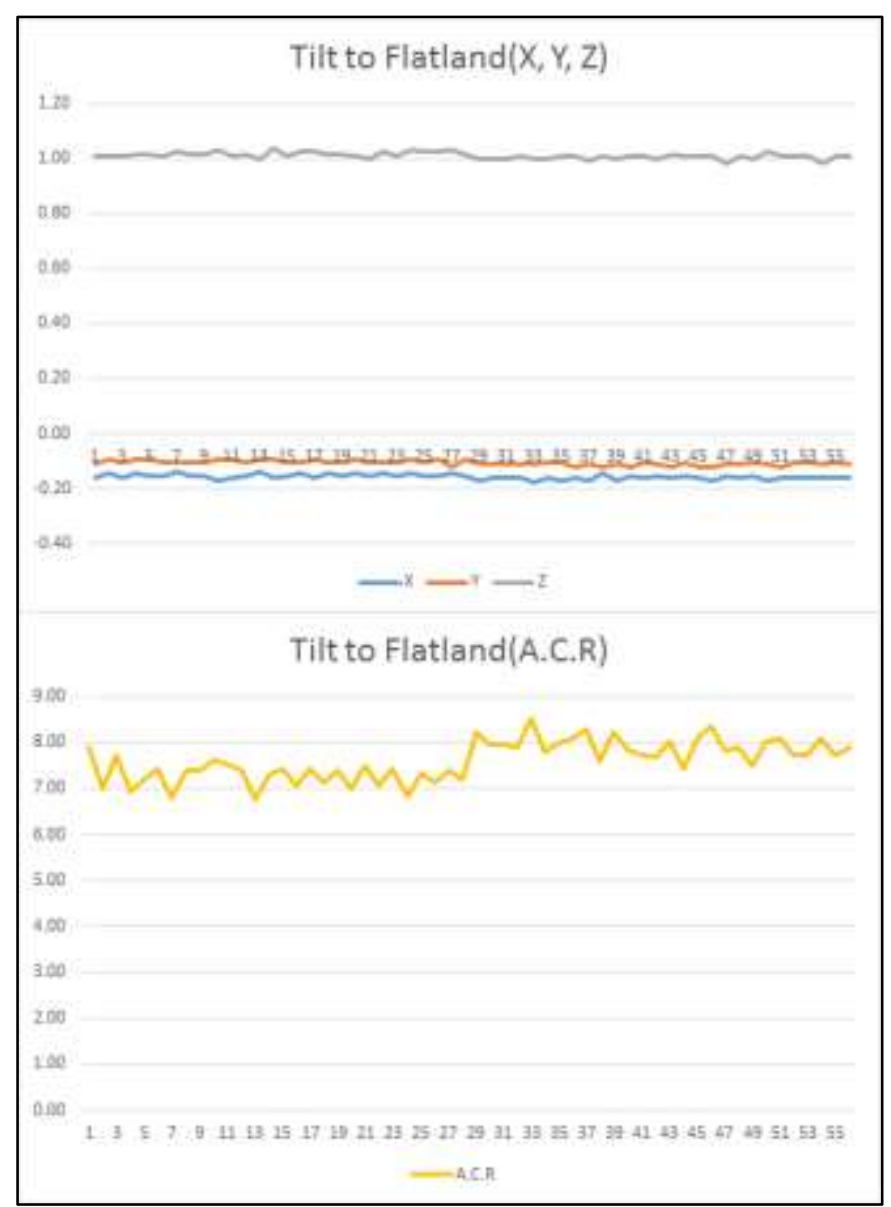

Figure 10. Parallel ACR Values

Figures 11 and 12 show the case of tilting backward and tilting forward. 


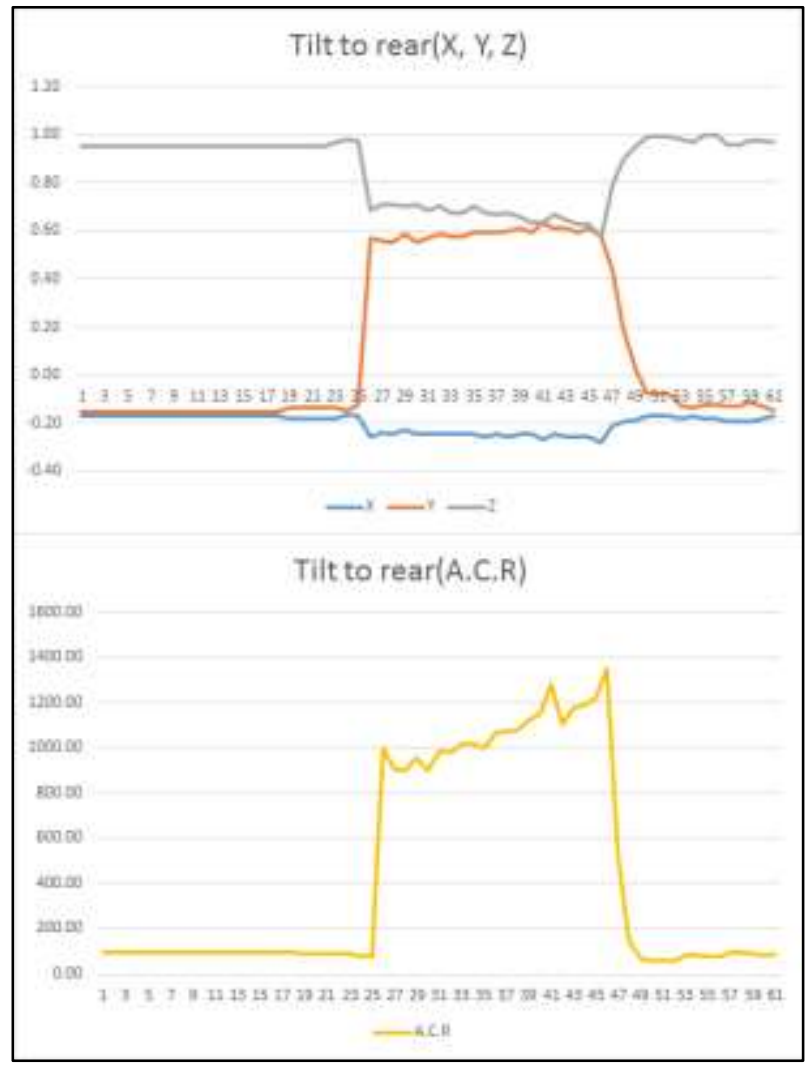

Figure 11. When Tilted Back

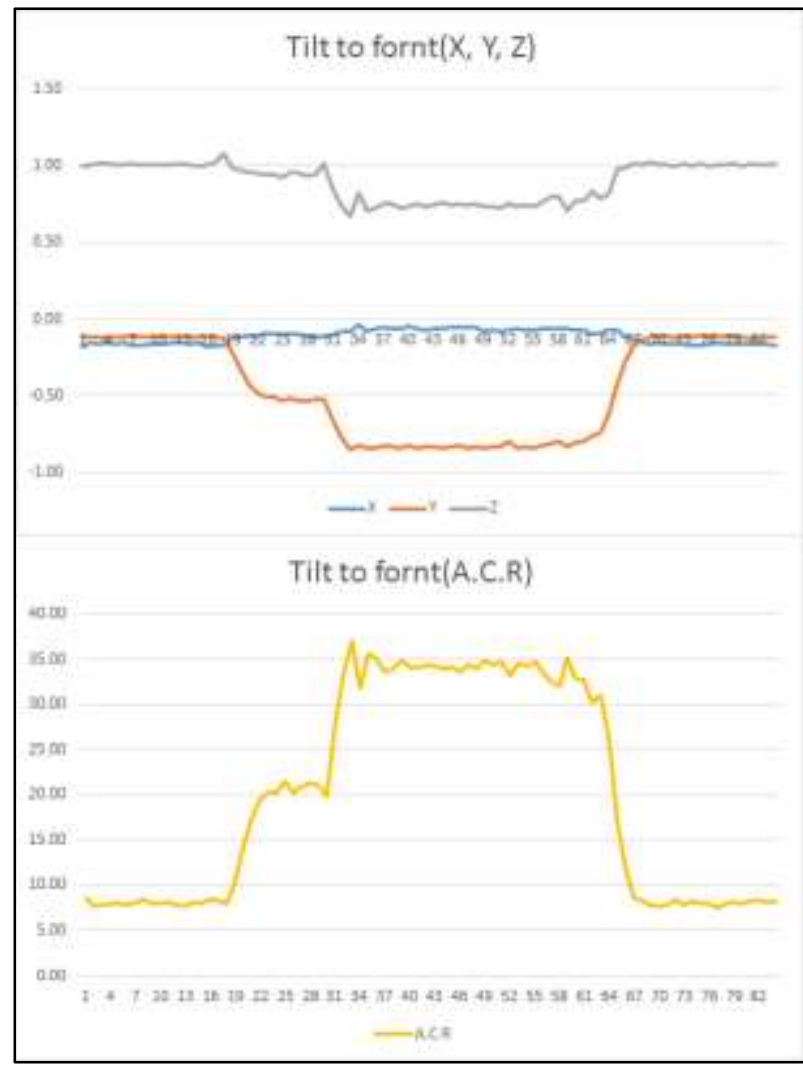

Figure 12. When I Lean Forward 
In Figures 11 and 12, the $\mathrm{X}, \mathrm{Y}$, and $\mathrm{Z}$ axes show significant fluctuations in the $\mathrm{Y}$ and $\mathrm{Z}$ axes. In the case of the $\mathrm{Y}$ axis, it can be seen whether it is tilted backward or tilted backward according to the amount of change in amount and the amount of change in sound. In case of ACR value, it is possible to see at a glance whether the SDD is inclined or not by changing only one of $\mathrm{X}, \mathrm{Y}$ and $\mathrm{Z}$ axis.

\section{Conclusion}

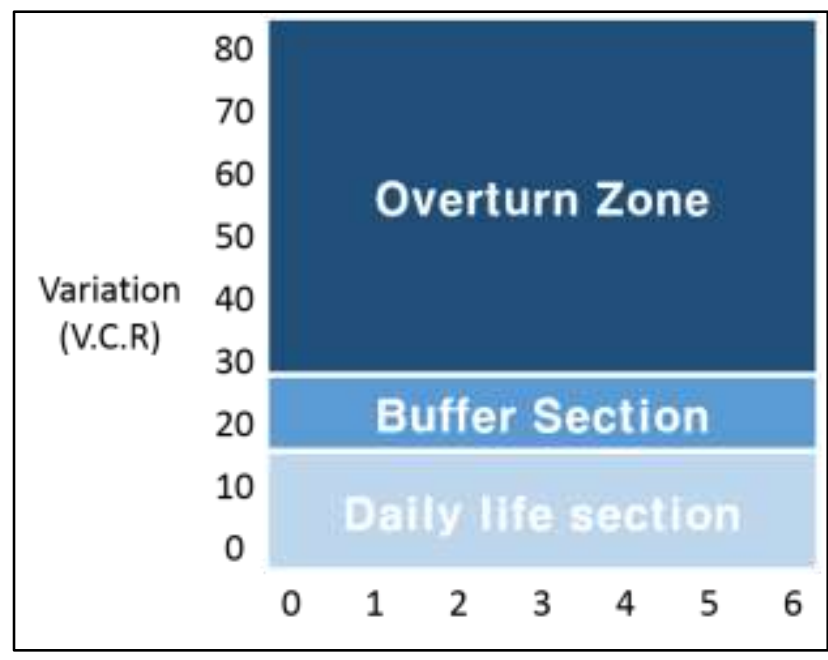

Figure 13. SSD Segment-specific Graphs

Figure 13 is a top display section based on the acceleration value ACR. On average, the measurement signal detected when moving in the daily life by a value 0 to 17 belong to the normal zone. 18 and 25 is a buffer region, The value is measured when the center of the wheelchair change in order to avoid falling or unsteady. After the 25 , falling zone, the values measured when fully wheelchair fell down.

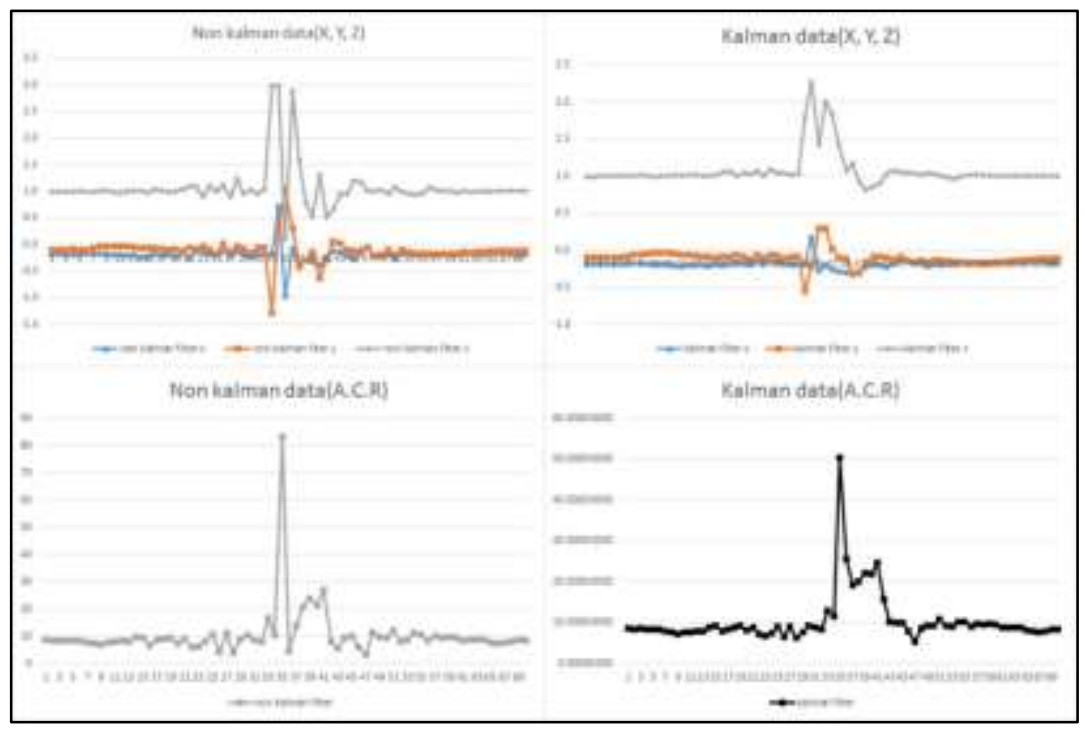

Figure 14. Acceleration and Inclination of ACR

Figure 14 is a graph of the ACR value obtained by measuring the acceleration and the inclination is measured in daily life. And the acceleration change amount can be sure that almost does not exceed the daily interval. Figure 15 will apply the Kalman filter in the 
ACR data, The rapidly changing ACR value that makes the output is stable. A section where the output using the Kalman filter, the resulting data as it passes through the bumps in the flat.

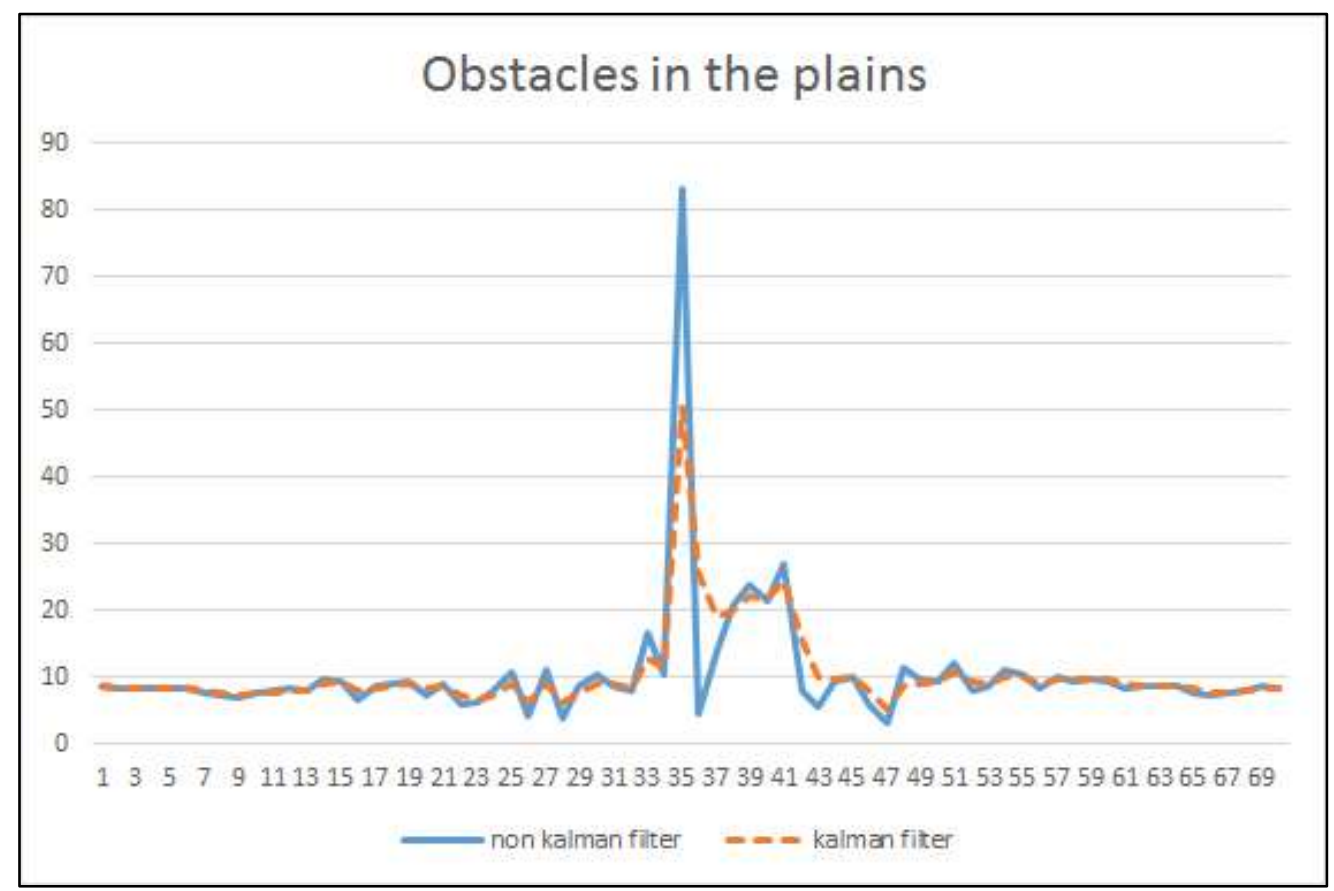

Figure 15. Comparison of the Non Kalman Filter Data and the Kalman Filter Data

\section{References}

[1] S. H. Park and J. S. Kim, "Optimal Power Maintain of Electric Wheelchair by using Applying Complementary Filter on the Smart Control System", Digital Contents Society Vol. 16, no. 3, (2015), pp. 355-363.

[2] H. D. Lee and H. D. Kim, "Vehicle crash and rollover detection sensor system", The Institute of Electronics Engineers of Korea vol. 20, no. 1, (2007), pp.101-102.

[3] J. H. Jang and J.A. Carriere, "A Research on the Dynamic Rollover Characteristics in View of Vehicle Dynamics", The Korean Society Of Automotive Engineers (2004), pp.734-740.

[4] A. Y. Jeon and G. R. Jeon, "Implementation of Falls Detection System Using 3-axial Accelerometer Senser", Journal of the Korea Academia-Industrial cooperation Society., vol 15, no. 1, (2010), pp. 15641572 .

[5] B. K. Jung, S. A. Lee and S. D. Min, "Development of Mobile Application based on ICF(International Classification of Functioning, Disability and Health) for Provision of Assistive Devices for People with Disabilities", Journal of the Korea Academia-Industrial cooperation Society., vol 15, no. 1, (2014), pp.377-385.

[6] H. I. Choi, J. S. Hong, K. J. Chun and C. W. Ko, "Biomedical analysis of lower limb's joint and analysis of IMU sensor during the fall", Journal of the Korea Academia-Industrial cooperation Society., vol 15, no. 1, (2014), pp.686-688.

[7] K. S. Kang, "The Effects of the Fall Prevention Exercise Program Focussed on Activity of daily living, Fear of fall and Quality of life for the Senior Citizen Center Elderly", Journal of the Korea AcademiaIndustrial cooperation Society., vol 17, no. 8, (2016), pp.267-272.

[8] J. S. Kong, "Design of an Electric Wheelchair Control Algorithm by Slope Recognition on uneven terrain", Journal of the Korea Academia-Industrial cooperation Society., vol 15, no. 9, (2014), pp.57385743.

[9] J. N. Kim, "Design of Electric Automatic Manual Wheelchair Driving System", Journal of the Korea Academia-Industrial cooperation Society., vol.14, no.11, (2013), pp.5392-5395.

[10] J. S. Kong, "Study on the Aid Control Algorithm for the Power-Assisted Smart Wheelchair", Journal of the Korea Academia-Industrial cooperation Society., vol. 12, no. 8, (2011), pp.3360-3365. 


\section{Acknowledgments}

Stars of this paper is supported by the Intramural Research Projects Hanseo University in 2016 and is presented at the International Mega-Conference on Green and Smart Technology held in Jeju Island, Korea from December 22 to 23, 2016, "Assured Data Device with Airports and Public Wheelchair Fall Prevention System" Is a revised and expanded paper.

\section{Authors}

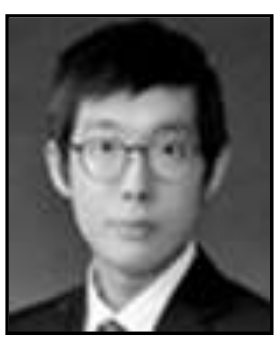

Seung-Hun Kim, He is a master's degree in Aeronautical System Engineering, Hanseo University. He graduated from the Department of Aeronautical and Mechanical Engineering, Hanseo University. He is studying Kalman filters, embedded systems, and smart network communications.

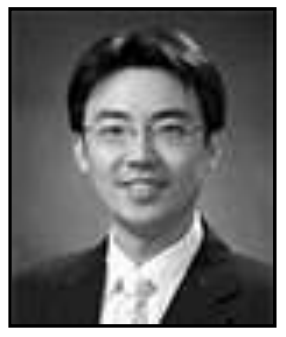

Min-Seok Jie, He graduated from the Ph.d degree in the department of avionics Engineering from Korea Aerospace University in 2006. he is Hanseo University Avionics Engineering Professor in to the present in 2008.

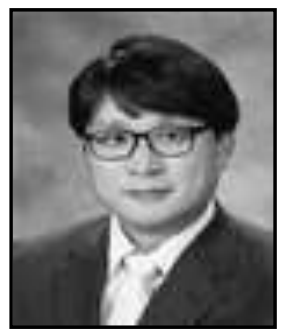

Won-Hyuck Choi (Corresponding author), He received the Ph.D. degree in avionics from Korea Aerospace University, Korea. He was a Professor of smart network at Doowon Technical University College, Korea. urrently, he is Professor of avionics at Hanseo University, Korea, where he has been since 2014. His present research interests include embedded system, home network. 
International Journal of Control and Automation Vol.10, No.7 (2017) 